\title{
Alcune considerazioni sull'ordinamento del corpus biologico di Aristotele
}

\author{
Giuseppe Feola*, Centro de Filosofia da Universidade de Lisboa
}

\section{Proposito}

In questo contributo tratterò di alcune classiche questioni circa l'ordinamento e la coerenza dottrinaria del Corpus Aristotelicum, limitando il problema al corpus psicobiologico.

Le questioni relative all'ordinamento sono le seguenti: qual è il rapporto tra le varie opere che lo compongono? Qual è il rapporto tra le singole parti di ciascuna opera? In che misura l'indice di ciascuna singola opera e del corpus nel suo insieme risale alla volontà ordinatrice di Aristotele, e in che misura invece l'eventuale lavoro di redattori posteriori ha sovraimposto all'opera di Aristotele criteri sistematici estranei alle sue intenzioni?

Le questioni relative alla coerenza dottrinaria sono queste altre: in che misura le dottrine esposte nelle varie opere (e nelle varie parti di ciascuna opera) si armonizzano tra loro? Come vanno spiegate eventuali incongruenze?

Si tratta di questioni che ho definito "classiche" perché appartengono al repertorio di domande che il XX secolo ha imparato a porre al corpus, e per

Il presente articolo è pubblicato nell'ambito del progetto di ricerca PRIN 2012: L'universalità e i suoi limiti: meccanismi di inclusione ed esclusione nella storia della filosofia enei dibattiti filosofici contemporanei (Unità locale di Pisa, Scuola Normale Superiore), finanziato dal Ministero dell'Università e della Ricerca. Desidero ringraziare Tiziano Dorandi per i suggerimenti bibliografici circa le vicende della biblioteca di Aristotele, e Maria Michela Sassi per la supervisione durante il lavoro di ideazione, stesura e correzione. Ove non altrimenti indicato, tutte le traduzioni dal greco, dal latino e da altre lingue straniere sono mie. 
rispondere alle quali si è addirittura originata una branca della bibliografia dotata di un suo specifico metodo e di sue particolari caratteristiche: una branca che si è sviluppata enormemente tra gli anni ' 20 e gli anni ' 80 del secolo scorso.

\section{Posizione del problema}

A legittimare il tipo di domande che ho appena ricordato è in primo luogo la trasmissione del corpus. Pochissimi corpora della grecità (lasciando da parte il caso particolarissimo di Omero) vedono la loro autorialità gravata da così tante ipoteche come quello di Aristotele. Lo studio ritenuto più autorevole sulla sua trasmissione nelle generazioni immediatamente successive alla morte dell'autore, quello di Paul Moraux ${ }^{1}$, evidenzia la totale incertezza in cui ci troviamo circala continuità della tradizione dopola morte di Aristotele. Moraux afferma ad esempio che già la seconda generazione di intellettuali successiva ad Aristotele (quella di Posidonio e di Panezio) potrebbe non aver avuto accesso agli originali delle opere di scuola ${ }^{2}$. Tuttavia, lo stesso Moraux, avvalendosi per lo più di procedimenti congetturali fondati sulla qualità, quantità ed esattezza delle menzioni di Aristotele da parte di autori di quest'epoca, ipotizza che esistessero copie variamente circolanti di singole opere, di varia qualità filologica: da quelle che potremmo considerare fedelissime, provenienti dalla biblioteca di Aristotele e appartenenti a esponenti della 'diaspora' del Peripato ${ }^{3}$, alle copie non autorizzate prodotte dal mercato librario per venire incontro alla curiosità intellettuale di collezionisti e pubblico colto.

1 P. Moraux, Der Aristotelismus bei den Griechen von Andronikos bis Alexander von Aphrodisia, Berlin-New York, Walter de Gruyter, 1973-1984; trad. it. di G. Girgenti, L'Aristotelismo presso i Greci, Milano, Vita e Pensiero, 2000.

2 Moraux, L'Aristotelismo, cit., vol. I, p. 20 n. 21.

3 Moraux, ibid., p. 20. Nelle pagine seguenti Moraux nota che, a causa di questa diaspora, già 50 anni dopo la morte di Aristotele ben quattro città avrebbero potuto vantare copie delle opere del Nostro provenienti dalla sua stessa biblioteca, o comunque copiate direttamente dagli originali: Atene (sede del Liceo), Scepsi (dove forse si trovavano quelle copie che Neleo, avendole ricevute per testamento da Teofrasto, non aveva venduto a Tolomeo d'Egitto), Alessandria (dove si trovavano quelle vendute a Tolomeo), Rodi (dove aveva fatto ritorno Eudemo). 
La fonte principale sulla sorte della biblioteca di Aristotele è Strabone, sulla veridicità della cui narrazione sono stati però avanzati forti dubbi. Strabone afferma che il figlio di Corisco, Neleo, dopo essere stato uditore di Aristotele e Teofrasto, ne avrebbe ereditato i libri e li avrebbe portati con sé a Scepsi ${ }^{4}$. Alla morte di Neleo, i suoi eredi, non interessati al sapere, tennero i libri in pessime condizioni; quando poi seppero che i re Attalidi cercavano libri per la costituzione della biblioteca di Pergamo, addirittura li nascosero in un fosso, dove furono maltrattati dall'umidità e dalle tarme $e^{5}$. Passò altro tempo, e i libri furono venduti al bibliofilo Apellicone di Teo, il quale avrebbe cercato di emendare maldestramente i testi corrotti dalle pessime condizioni di conservazione, mettendo così in circolazione libri pieni di errori. Quando Silla conquistò Atene, confiscò la biblioteca di Apellicone; di essa si curò il grammatico Tirannione; anche lui, però, non si sarebbe dimostrato (a giudizio di Strabone) all'altezza del compito (Geografia, XIII, 1, 54, 608-609).

E molte vicissitudini ancora interverranno, prima di giungere all'intervento di Andronico di Rodi, che per primo (secondo la ricostruzione di Plutarco, Vita di Silla, 26) ${ }^{6}$ stabilisce un corpus riconosciuto, grazie al quale

4 E qui emerge il primo nodo problematico: Stratone, il successore di Teofrasto, avrebbe mai permesso a Neleo di portar via le copie delle opere di Aristotele, se il Liceo non ne avesse avute di altre? La difficoltà è sollevata da J. Barnes, Roman Aristotle, in Philosophia togata II. Plato and Aristotle at Rome, a cura di J. Barnes, M. Griffin, Oxford, Clarendon Press, 1997, p. 14. Anche secondo H.J. Drossaart-Lulofs (Neleus of Scepsis and the Fate of the Library of the Peripatos, in Les textes philosophiques et scientifiques grecs au Moyen Age latin. Hommage à Fernand Bossier, a cura di R. Beyers, J. Brams, D. Sacré, K. Verrycken, Leuven, Leuven University Press, 1999, pp. 9-24) è lecito supporre che, se pure il testamento di Teofrasto avesse previsto il lascito di tutta la biblioteca a Neleo, è a dir poco implausibile che Stratone lasciasse partire la biblioteca per Scepsi senza opporsi: avrebbe ad esempio potuto ricorrere all'aiuto di uno dei moltissimi avvocati che in Atene erano specialisti nell'impugnare testamenti.

5 A questo elemento della storia Barnes è propenso a credere, proprio perché inverosimile. Barnes argomenta che, in generale, chi inventa storie allo scopo di essere creduto tende a inventare storie verisimili, e la nostra storia non lo è: perché mai i successori di Neleo avrebbero dovuto seppellire i libri, piuttosto che venderli a caro prezzo al re di Pergamo? (Barnes, Roman Aristotle, cit., p. 8). Ciò che Barnes nega, dunque, non è la veridicità della storia qui narrata; bensì che le copie di cui è questione in questa storia, e che furono sotterrate e rovinate dalle tarme, fossero tutte le copie disponibili (ibid., p. 14).

6 Cfr. Moraux, L'Aristotelismo, cit., vol. I, pp. 53-56. Barnes, Roman Aristotle, cit., p. 24, data la pubblicazione del lavoro di Andronico a dopo la morte di Cicerone (43 a.C.), 


\section{- almeno secondo l'opinione più diffusa ${ }^{7}$ - il Peripato, prima in declino, riconquista un posto di primo piano tra le scuole filosofiche ${ }^{8}$.}

in base al fatto che non ne troviamo menzione nelle opere e nell'epistolario dell'Arpinate, sempre attento alle novità bibliografiche. La datazione proposta da Barnes è chiaramente volta a ridimensionare l'importanza del lavoro di Andronico nel processo di diffusione delle opere esoteriche, visto che, se da un lato Cicerone non menziona Andronico, dall'altro però conosce e menziona una distinzione tra opere esoteriche ed essoteriche (De finibus bonorum et malorum, V, 5, 12). Secondo J. Dillon, The Reception of Aristotle in Antiochus and Cicero, in Brill's Companion to the Reception of Aristotle in Antiquity, a cura di A. Falcon, Leiden-Boston, Brill, 2016, pp. 183-201, le opere esoteriche tornarono nel circuito librario proprio durante la vita di Cicerone, il quale, avendo accesso alla biblioteca di Silla, avrebbe potuto conoscerle e sfogliarle, ma, avendo in precedenza ammirato l'Aristotele dei dialoghi come maestro di stile, sarebbe in qualche modo rimasto deluso dalla sciatteria dell'esposizione di queste nuove opere, al punto da trascurarle completamente (ibid., p. 186): ciò spiegherebbe come mai le menzioni ciceroniane delle opinioni aristoteliche mostrino così poche somiglianze con le dottrine a noi familiari. Dillon esamina svariate menzioni di Aristotele nel corpus Ciceronianum, mostrando che in nessun caso abbiamo la certezza di avere a che fare con le opere a noi note, mentre viceversa in molti casi la discrepanza tra le dottrine delle opere esoteriche e quelle riportate da Cicerone è palese. La datazione proposta da Dillon per il ritorno in circolazione delle opere esoteriche è coerente coi racconti di Strabone e Plutarco.

7 Una voce fuori dal coro è Barnes, Roman Aristotle, cit., il quale attribuisce scarsissima importanza al lavoro di Andronico, a suo avviso molto maldestro: «I suspect that Andronicus' textual activity aroused little interest among Aristotelian scholars and left little mark on Peripatetic scholarship. [...] Andronicus merely published copies of corrupt manuscripts» (pp. 30-31; cfr. anche p. 33). E ancora: «There is no reason to think that the Peripatetic renascence was any more dependent on books; and there is no reason to think that Andronicus played midwife at the rebirth» (ibid., p. 66). Mi sembra però che Barnes avrebbe dovuto fornire una spiegazione alternativa di questa coincidenza cronologica tra l'operazione andronicea e il rifiorire del Peripato, piuttosto che limitarsi a negarne la correlazione. Una posizione più equilibrata è, forse, quella di M. Hatzamichali (Andronicus of Rhodes and the Construction of the Aristotelian Corpus, in Falcon (a cura di), Brill's Companion, cit., pp. 81100): sarà pur vero che varie copie di singole opere dell'Aristotele esoterico circolavano già in età ellenistica; ma Andronico avrebbe reso disponibili al pubblico, in una veste unitaria e in un ordinamento sistematico, lavori che prima circolavano poco e 'in ordine sparso', e che perciò erano di difficile reperimento e di ancor più difficile consultazione. Anche se è esagerato dire che Andronico fece riemergere l'Aristotele esoterico dall'oblio, l'immissione sul mercato di copie nuove in un ordine sistematico sarebbe appunto - secondo Hatzamichali - quel fattore che può spiegare l'improvvisa crescita dell'interesse per Aristotele a partire dal I sec. a.C. e soprattutto la grande differenza tra il catalogo laerziano e quello di Tolomeo (cfr. infra nota 11).

8 Cfr. Moraux, L'Aristotelismo, cit., vol. I, p. 57. Interessante l'osservazione di D. Lefebvre, Aristotle and the Hellenistic Peripatos: From Theophrastus to Critolaus, in Falcon (a cura di), Brill's Companion, cit., pp. 13-34, secondo cui la lettura dei cataloghi delle opere degli immediati successori di Stratone alla guida del Peripato dà l'impressione che questi filosofi non conoscessero le opere di Aristotele a noi note (p. 29). 
Quali che siano state queste vicissitudini, quali che siano stati i caratteri dell'intervento di Andronico, ciò che è importante per i nostri scopi in questa sede non è stabilire la portata del suo intervento, bensì la netta differenza che si può notare tra il catalogo pre-androniceo che troviamo in Diogene Laerzio e quelli post-andronicei': nel primo gli scritti di scuola compaiono in scarso numero $^{10}$, nel secondo compaiono massicciamente, e già con titoli tali da

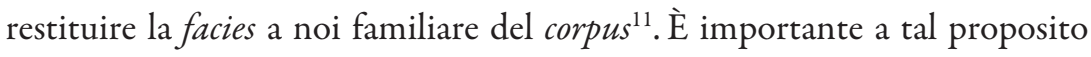
una notizia che ci dà Porfirio, paragonando il proprio lavoro di editore di Plotino a quello di Andronico:

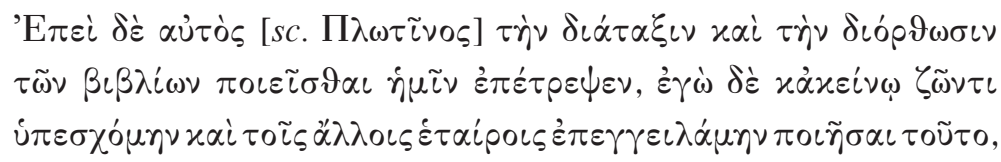

9 Cfr. Moraux, L'Aristotelismo, cit., vol. I, pp. 68-69. I cataloghi delle opere di Aristotele sono riportati in V. Rose, Aristotelis qui ferebantur librorum fragmenta, editio stereotypa editionis primæ (MDCCCLXXXVI), Stuttgart, Teubner, 1966, pp. 1-22. Essi sono tre: quello di Diogene Laerzio (Vite dei filosofi, V, 21-27), quello di Esichio, e quello di Tolomeo.

${ }^{10}$ Se dunque è vero che la lista di Diogene riflette i pinakes delle biblioteche di Pergamo e Alessandria (come suppone T. Dorandi, Diogene Laerzio e la tradizione catalogica. Liste di libri nelle Vite e opinioni dei filosofi, in «Antiquorum philosophia», VII (2013), pp. 107-126, e in particolare p. 123), allora dovremmo inferirne che nelle principali biblioteche del mondo ellenistico circolasse un corpus la cui facies era molto diversa da quella a noi familiare. Barnes, Roman Aristotle, cit., pp. 43-44 e p. 54, suggerisce che la differenza tra il corpus noto al pubblico ellenistico e quello post-androniceo rifletta solo il fatto che fino ad Andronico i medesimi libri che poi sarebbero comparsi nel nostro corpus circolassero già, ma come trattazioni separate, e dunque con titoli diversi da quelli con i quali noi li conosciamo; nel seguito dell'articolo Barnes ridimensiona ulteriormente la portata dell'intervento di Andronico.

${ }^{11}$ Se avessimo solo il catalogo di Diogene Laerzio sarebbe per noi impossibile congetturare che Aristotele abbia scritto opere in qualche modo afferenti al corpus quale noi lo conosciamo, laddove, almeno in alcune sezioni del catalogo di Esichio, il Corpus Aristotelicum è già in qualche modo riconoscibile: in particolare è riconoscibile (cfr. la sez. 155-159) il blocco HA-MA-PA-GA. Sulla difficile questione delle liste delle opere di Aristotele, e sui problemi posti dal loro reciproco confronto, cfr. Dorandi, Diogene Laerzio, cit., p. 116: «Qualunque sia la fonte del catalogo delle opere di Aristotele che Diogene trasmette - se pure mai potrà essere determinata - quello che resta evidente e indiscutibile è il fatto che, all'epoca in cui questa lista venne redatta, alcuni libri di differenti trattati circolavano separati come unità indipendenti, che molte opere avevano titoli diversi da quelli con i quali le conosciamo oggi e che mancava inoltre una divisione delle opere in gruppi tematici». 


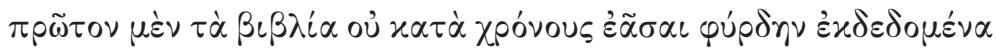

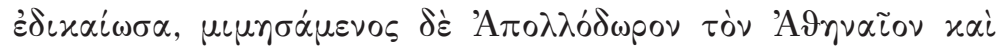

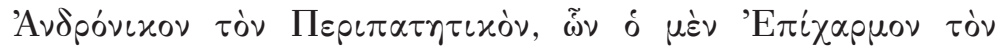

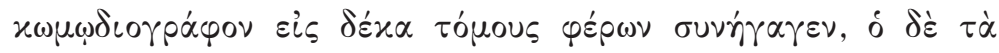

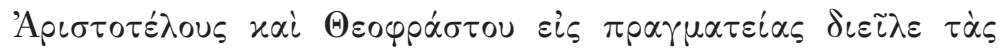

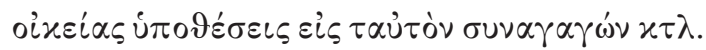

Poiché egli stesso [sc. Plotino] mi aveva incaricato di effettuare la disposizione e la correzione dei [suoi] libri, ed io stesso a lui ancora vivo l'avevo promesso, e avevo annunciato agli altri compagni che l'avrei fatto, anzitutto ritenni giusto licenziare i libri (che erano stati prodotti [da Plotino] alla rinfusa) non in base ai tempi [di composizione], bensì imitando Apollodoro di Atene e Andronico il Peripatetico, di cui il primo raccolse le opere del commediografo Epicarmo riportandole in dieci libri, mentre il secondo divise in trattati le opere di Aristotele e Teofrasto riunendo in un'identica rubrica gli argomenti affini etc. (Porfirio, Vita di Plotino, 24, 2-12, trad. mia).

Porfirio afferma di aver fatto, per le opere di Plotino, quel che Andronico avrebbe fatto per quelle di Aristotele: distribuire le opere in pragmatie, mettendo insieme le trattazioni di argomento affine ${ }^{12}$. Il passo

12 Barnes, Roman Aristotle, cit., passim, affronta anche la questione se quest'opera di riordinamento diede luogo a opere la cui costituzione interna era sensibilmente diversa da quella precedente, o se invece Andronico si limitò a indicare un ordine preferenziale di lettura per opere i cui indici erano già quelli che troviamo nella tradizione posteriore. Il paragone con ciò che Porfirio fece con le opere di Plotino spinge Barnes verso la seconda soluzione: «Porphyry says nothing at all about the creation of our Aristotelian treatises - he does not say that Andronicus invented Top. or put together $E N$ or cobbled up Met. Nor he does imply such creative activity. His own work on Plotinus' manuscripts did not involve the creation of 'new' works. Porphyry did not take an essay here and a squib there and unite them into a tract or treatise. On the contrary, he found fiftyfour essays and he left fifty-four essays. He found the fifty-four essays in no order than the order of their writing, and he left the same fifty-four essays in what he took to be a more satisfactory and a more philosophical order» (ibid., pp. 39-40). Questo punto dell'argomentazione di Barnes non mi sembra convincente: il parallelo che Porfirio sta istituendo potrebbe essere benissimo tra i singoli trattati di Plotino (da lui ordinati in Enneadi) e i singoli libri di Aristotele che, in base a questo parallelismo, Andronico avrebbe ordinato in trattazioni più ampie; e, in tal caso, la testimonianza di Porfirio starebbe implicando proprio ciò che Barnes nega: che fu Andronico a 'cucire insieme' quelle trattazioni più ampie quali p.es. i Topici, la Fisica o la Metafisica, a partire dai singoli libri. 
di Porfirio sembra infatti implicare che fu Andronico a creare per esempio i Topici o la Fisica, mettendo assieme libri che prima circolavano come opere indipendenti. Questa ricostruzione ha il vantaggio di esser coerente con la differenza - che già abbiamo riscontrato ${ }^{13}$ - tra i cataloghi pre- e quelli post-andronicei. Se dunque questa informazione dataci da Porfirio è affidabile, ad Andronico dovrebbe risalire l'ordinamento delle opere aristoteliche.

Se ora proviamo a estrarre un sunto da questa messe confusa di dati e di opinioni, vediamo che di certo vi è molto poco: un'eclissi dell'interesse per Aristotele nei 250 anni successivi alla sua morte e una tendenza ad attribuirgli dottrine diverse da quelle a noi note; un'improvvisa crescita dell'interesse per lui nel I sec. a.C., in concomitanza con le vicende narrate da Strabone e Plutarco e con l'opera di Andronico, di cui però sappiamo poco o nulla. Alla luce di ciò, il parere di Hatzamichali, secondo cui Andronico, pur senza produrre una vera e propria edizione critica, sarebbe il responsabile dell'ordinamento e della reimmissione nel circuito librario delle opere esoteriche, che prima circolavano in scarso numero e in ordine sparso, $\mathrm{mi}$ sembra condivisibile ${ }^{14}$.

Eccoperchéèlegittimo porsilaquestionese (ein chemisura)l'ordinamento androniceo del corpus rispecchi le intenzioni di Aristotele (ammesso che Aristotele avesse delle precise intenzioni riguardo all'ordinamento delle sue opere). Si noti infatti che Andronico vive in un'epoca nella quale, proprio dopo la svolta nell'organizzazione e nell'esposizione del sapere segnata da Aristotele, la forma del trattato sistematico è ormai divenuta la forma paradigmatica della comunicazione del sapere scientifico e filosofico, cosa che certo non può ancora dirsi della prima metà del IV sec. a.C., quando l'esposizione della scienza e della filosofia avveniva per lo più tramite la forma dello scritto breve oppure del dialogo.

\footnotetext{
13 Cfr. supra, nota 12 .

${ }^{14}$ Hatzamichali, Andronicus of Rhodes, cit., passim.
} 
Se, da un lato, non è più molto frequente vedere proposte ermeneutiche simili a quelle di Jæger ${ }^{15}$ e Nuyens ${ }^{16}$, che si sentivano in grado di stabilire quali capitoli e capoversi della tale o tal altra opera andassero assegnati a quale periodo, di discutere della successione cronologica di singole frasi che differiscono tra loro solo per diverse sfumature nella presentazione lessicale dello stesso concetto, e di ricostruire la biografia intellettuale di Aristotele sulla base di questi indizi, non è però più nemmeno possibile (ed è questo il guadagno sostanziale della prospettiva jægeriana) assumere ingenuamente che il corpus sia espressione di una dottrina unitaria e coerente dal punto di vista dei contenuti: è certo lecito sostenere questa opinione, ma bisogna argomentarla; meno ancora è possibile assumere che il corpus sia la manifestazione di un insegnamento monolitico dal punto di vista dell'esposizione, simile alle trattazioni sistematiche, accuratamente pianificate anche dal punto di vista dell'architettonica generale, proposte da autori moderni come Spinoza o Cartesio: ancora una volta, è possibile sostenerlo, ma bisogna argomentarlo.

In questo quadro è normale che appaiano proposte radicalmente opposte tra loro, come vedremo subito affrontando finalmente i problemi specifici posti dalle opere biologiche. Ad esempio, Vegetti e Lanza affermano che la Historia animalium apparterrebbe a una fase del pensiero scientifico di Aristotele i cui principii sarebbero stati sconfessati dagli sviluppi successivi della sua metafisica, e che il corpus biologico sarebbe nato dalla saldatura artificiale tra i due blocchi, di diversa impostazione epistemologica, costituiti l'uno dalla Historia, l'altro dal De partibus e dal De generatione animalium ${ }^{17}$.

15 W. Jæger, Aristoteles. Grundlegung einer Geschichte seiner Entwicklung, Berlin, Weidmannsche Buchhandlung, 1923; trad. it. di G. Calogero, Aristotele. Prime linee di una storia della sua evoluzione spirituale, con aggiunte e appendice dell'Autore, Firenze, La Nuova Italia, 1935, rist. 1968.

${ }^{16}$ F. Nuyens, Ontwikkelingsmomenten in de zielkunde van Aristoteles, Njimegen-Utrecht, Dekker \& Van de Vegt, 1939; trad. fr. di T. Schillings, L'évolution de la psychologie d'Aristote, Louvain, Insitut Supérieur de Philosophie, 1948.

${ }^{17}$ D. Lanza, M. Vegetti, Aristotele. Opere biologiche, Torino, UTET, 1971, rist. 1996; cfr. in particolare il breve saggio Origini e metodi della zoologia aristotelica nella Historia 
Viceversa, Lennox insiste alquanto sul ruolo di ciascun trattato nell'insieme organico del corpus, giungendo, in nome di tale organicità, a sposare un'ipotesi a suo tempo proposta da Balme ${ }^{18}$, e a rovesciare l'ipotesi abbastanza diffusa della priorità temporale della Historia rispetto al De partibus: la Historia contiene un maggior numero di osservazioni naturalistiche rispetto al De partibus, e costituirebbe l'implementazione empirica del programma schizzato nel De partibus ${ }^{19}$.

Nell'affrontare la questione, nelle prossime pagine mi volgerò ai due seguenti problemi: (1) qual è il rapporto che intrattengono le singole opere biologiche tra loro? (2) qual è il rapporto del corpus biologico col De anima?

\section{Opinioni autorevoli circa il rapporto tra le varie opere del corpus biologico}

Per quanto riguarda i rapporti interni al corpus biologico, circa i quali abbiamo già menzionato le opinioni di Vegetti e Lanza e di Lennox, sarà ora il caso di vedere cosa ne dice il fondatore dell'approccio 'evolutivo' al pensiero di Aristotele, ciò̀ Jæger. Jæger non dedica al corpus psicobiologico un capitolo specifico nel suo trattato: menziona le opere biologiche solo per asserire, come se si trattasse di un corollario ovvio della sua argomentazione principale, che opere così piene di dettagli empirici devono necessariamente essersi originate nel momento in cui Aristotele era massimamente lontano dallo spirito dell'Accademia platonica, e cioè alla fine della sua vita ${ }^{20}$. Jæger apporta anche argomentazioni più circostanziate per una datazione tarda

Animalium (ibid., pp. 77-128): secondo Vegetti e Lanza, la Historia animalium, concepita in origine come summa generale del sapere di Aristotele in campo biologico, sarebbe stata poi degradata dall'autore a mera raccolta e collezione di dati, quando l'articolarsi sempre maggiore dell'approccio ilomorfico e della tematica della potenza-atto avrebbe imposto l'elaborazione di una nuova biologia: quella del De partibus e del De generatione (cfr. ibid., p. 82).

${ }^{18}$ D.M. Balme, The Place of Biology in Aristotle's Philosophy, in Philosophical Issues in Aristotle's Biology, a cura di A. Gotthelf, J.G. Lennox, Cambridge-New York, Cambridge University Press, 1987, pp. 9-20.

${ }_{19}$ J.G. Lennox, Aristotle. On the Parts of Animals, Oxford, Clarendon Press, 2001, rist. 2004, Introduction, p. xiv.

${ }^{20}$ Cfr. Jæger, Aristotele, cit., pp. 417-418 e 448. 
delle opere biologiche: per esempio il fatto che le notizie sugli animali esotici contenute nella Historia animalium sarebbero potute giungere in Grecia solo tramite la spedizione di Alessandro ${ }^{21}$. Per quanto riguarda le relazioni interne al corpus biologico, Jæger ipotizza che la Historia stesse alle opere più speculative nel medesimo rapporto in cui la raccolta delle Costituzioni stava alla Politica: si tratterebbe di raccolte di dati, che Aristotele avrebbe delegato a più persone; in tal modo si spiegherebbero le incongruenze che di tanto in tanto è possibile rinvenire nell'esposizione ${ }^{22}$. È poi notorio lo iato che Jæger crede di individuare tra il III libro del De anima e tutto il resto della psicologia di Aristotele: il III libro sarebbe un relitto della fase platonica del pensiero di Aristotele ${ }^{23}$.

Si noti che Jæger non nega che sia ammissibile anche un ordine sistematico di lettura del corpus; egli però afferma che tale ordine restituirebbe solo l'ordine che Aristotele, alla fine della sua carriera, assegnò al corpus, e non quello - a suo avviso più significativo - in cui l'autore compose le sue opere ${ }^{24}$. Se da un lato Jæger ha di certo ragione nell'affermare che è arbitrario (e anche ingenuo) presumere che l'ordine di composizione debba rispecchiare quello indicato come preferibile per la lettura sistematica, dall'altro la giustificazione del fatto che l'ordine sistematico (quale che sia) dovrebbe essere meno significativo di quello cronologico di composizione sta tutta in una intuizione che Jæger ritiene di avere circa la natura dello spirito di Aristotele: Jæger considera del tutto estraneo allo spirito di Aristotele qualsiasi tentativo di cercare o costruire un'architettura generale del sapere; la precedenza, almeno nel tardo Aristotele, sarebbe data sempre al particolare ${ }^{25}$, e l'importanza

${ }^{21}$ Ibid., p. 330.

${ }^{22}$ Ibid., p. 448: «È ormai difficile stabilire esattamente quale parte abbia avuta di persona Aristotele nella redazione della Storia degli animali».

${ }^{23}$ Ibid., pp. 451-452.

${ }^{24}$ Ibid., p. 398: «Quel che si ottiene in tal modo è, nel migliore dei casi, solo la successione prevista dallo stesso Aristotele, al termine della sua attività letteraria, dal punto di vista pedagogico e contenutistico».

${ }^{25}$ Ibid., pp. 510-511: «Ciò accade per la Storia degli animali non altrimenti che per la Metafisica e la Politica. Abbozzi di ordinamento sistematico, spesso inseriti solo durante il 
delle ricerche del tardo Aristotele starebbe nell'aver dato l'avvio a una nuova stagione dello spirito greco, quello della scienza ellenistica; quindi: nessuna architettonica generale del sapere (se non a un livello molto estrinseco), e dunque nessun ordine sistematico delle opere. L'unico ordine che conta è quello genealogico, che Jæger crede di aver scoperto.

Com'è noto, Nuyens tentò di estendere al corpus psicobiologico il metodo jægeriano, giungendo a una diversa cronologia relativa delle opere del Nostro: le opere biologiche, nella misura in cui manifestano la tendenza a descrivere la relazione anima-corpo mediante la metafora 'artigianostrumento' (concezione da Nuyens chiamata "strumentalismo vitalistico"), apparterrebbero non alla fase più tarda dell'attività di Aristotele, ma ad una intermedia tra il (presunto) dualismo giovanile e l'ilomorfismo 'compiuto' di De anima $\mathrm{II}^{26}$.

In un contributo della metà degli anni '80 D.M. Balme ha riproposto (ma in modo più minimalista di quanto fosse consueto nell'approccio jægeriano, e senza pretese di disegnare l'intero sviluppo intellettuale dell'autore), l'idea che vi sia stato uno sviluppo del pensiero di Aristotele, mettendone al centro l'impatto che sulle sue concezioni metafisiche avrebbero avuto gli studi biologici ${ }^{27}$. Balme osserva che il De partibus animalium dispiega una descrizione degli animali per classi, cioè per specie, mentre il De generatione animalium si concentra sulla trasmissione del patrimonio genetico individuale ${ }^{28}$. Il cambiamento di approccio sarebbe stato dovuto alla scoperta, avvenuta al momento di lavorare alla Historia animalium, di differenze che stanno, nell'albero classificatorio, al di sotto del livello della specie, e così fini (secondo Balme) da poter giungere a definire l'individuo: Balme osserva che la teoria della trasmissione del patrimonio genetico fornita nel De generatione

posteriore lavoro di fusione, restano senz'alcuna applicazione o vengono applicati solo a metà. Quella della sistemazione esteriore non è stata un'idea originaria di questo architetto, e quindi neppure tale sistema si può 'ricostruire'».

${ }^{26}$ La tesi è esposta diffusamente in Nuyens, L'évolution, cit., passim.

${ }^{27}$ Balme, The Place, cit.

${ }^{28}$ Ibid., p. 12. 
animalium, di fatto, suppone che vi sia una forma individuale, ulteriormente specificata rispetto a quella della specie: che si tratti di forme individuali o di forme sub-specifiche ${ }^{29}$, resta il fatto che $G A$ propone, sulla base di ricerche empiriche, una soluzione a una questione sulla quale, invece, Metafisica VII si dibatte in discussioni dialettiche che non portano a risultati conclusivi ${ }^{30}$. Quindi la Historia e il De generatione sarebbero posteriori a Metaph. VII ${ }^{31}$. Viceversa, De partibus II-IV, con le sue considerazioni assiologiche che ricorderebbero quelle del Timeo, dovrebbe essere anteriore, addirittura del periodo accademico ${ }^{32}$. Balme può sostenere questa ricostruzione perché afferma, en passant, che le informazioni empiriche contenute nel Departibus non sarebbero più accurate di quelle a disposizione di qualunque persona colta, e che il vero salto nella raccolta dei materiali Aristotele l'avrebbe compiuto solo con la Historia ${ }^{33}$. L'ordine di scrittura proposto da Balme è dunque: (1) $P A$ II-IV e $I A$ al tempo dell'Accademia; (2) $P A$ I al tempo di Physica II e Metaph. VII, pure nel periodo accademico ma dopo i trattati prima menzionati; (3) $P N$ alla fine del periodo accademico; (4) $H A$ iniziato nel periodo accademico ma continuato durante i viaggi ${ }^{34}$; (5) $G A^{35}$.

Come si vede, la ricostruzione di Balme si fonda su due assunti non proprio pacifici: (I) che tanta parte dell'opera di Aristotele (inclusa la grande elaborazione della metafisica della sostanza, fondamentale per l'impostazione del Departibus) risalga al periodo accademico, (II) che le informazioni zoologiche, anatomiche, embriologiche, del Departibus e del De generatione non richiedessero una ricerca

${ }^{29}$ Da qui in poi parlerò, a questo proposito, di forme "sub-specifiche" e non "individuali", perché (a) ai fini della presente discussione basta ipotizzare che Aristotele sia giunto a porre delle forme sub-specifiche; $(b)$ credo che le forme individuali pongano dei problemi ulteriori, che è meglio non sollevare qui.

${ }^{30}$ Ibid., pp. 18-20.

31 Ibid., p. 11.

32 Ibid., p. 17.

33 Ibid., p. 16.

${ }^{34}$ Ibid., p. 13. HA costituirebbe il punto di riferimento databile, visto che, com'è noto, moltissimi dati lì esposti si riferiscono ad animali tipici della zona di Lesbo, in particolare della famosa laguna di Pyrrha, oggi Kalloni, e a Lesbo Aristotele visse tra il 344 e il 342 a.C.

35 Ibid., pp. 17-18. 
specifica, sul campo, che superasse in dettaglio e accuratezza le informazioni già disponibili mediante la così detta 'cultura generale'.

Questo secondo assunto, in particolare, mi sembra insostenibile.

Secondo Balme, al momento di redigere la Historia, Aristotele vi avrebbe inserito un riassunto schematico delle informazioni già raccolte nelle altre due opere, e vi avrebbe aggiunto quelle raccolte di persona, alcune delle quali sarebbero anche in conflitto con le teorie espresse nei trattati preesistenti ${ }^{36}$. La Historia sarebbe, nella ricostruzione di Balme, un work-in-progress, un laboratorio di appunti iniziato a Lesbo, e che andò crescendo per tutta la durata della vita di Aristotele, e alla quale non fu mai data l'ultima mano ${ }^{37}$. Perciò l'ordinamento enunciato in $H A$ 491a7-14, che pone $H A$ prima di $P A$ e $G A$, si riferirebbe invece a un ordine di lettura stabilito a scopo didattico in una fase successiva, nella quale sarebbero stati anche aggiunti i riferimenti incrociati, i quali, sia dal punto di vista del contenuto sia da quello della sintassi, possono esser rimossi senza alterare l'ordine dell'esposizione. Ad avviso di Balme, infine, informazioni più dettagliate sull'ordine di lettura consigliato da Aristotele sono per noi inaccessibili, a causa dell'opera di riedizione di Andronico, che avrebbe stabilito l'archetipo dei manoscritti che abbiamo, dopo tutte le vicissitudini narrate in Strabone (al cui racconto Balme ritiene si possa dar credito $)^{38}$.

Ci limiteremo qui a rilevare che, visto che la correlazione tra la strutturazione del vivente delineata nel Departibus animalium e l'ontologia sviluppata in Metaph. VII-IX sembra evidente al di là di qualunque dubbio, è chiaro che le proposte di Balme non potranno trovare accoglienza in chi non sia disposto ad ammettere una datazione così alta di lavori (i libri centrali della Metafisica) normalmente considerati rappresentativi del pieno sviluppo filosofico dello Stagirita. Ricordiamo fra l'altro che Furth ha addirittura

\footnotetext{
${ }^{36} \mathrm{Ibid}$., pp. 13-15. Esempi assortiti di luoghi in cui le osservazioni raccolte nella Historia confliggerebbero con le teorie del De partibus e del De generatione sono esposti alle pp. 14-15.

${ }^{37}$ Ibid., p. 17.

${ }^{38}$ Ibid., pp. 12-13.
} 
proposto un'analisi dell'ontologia di Aristotele interamente incentrata sull'idea che Metaph. VII-IX sia una discussione formale sui principii della biologia e su come si debba concepire il che cos'è di un organismo, in particolare di un animale ${ }^{39}$, idea che trovo tutt'altro che stravagante, e anzi notevolmente centrata.

\section{Stato dell'arte}

Mentrelaquestionedellacronologia relativadellacomposizionedellediverse opere sembra rimanere irresolubile, circa la questione della corrispondenza tra le intenzioni di Aristotele e l'indice del corpus (quale è possibile ricostruirlo dai richiami incrociati tra le diverse opere, e in particolare dalle dichiarazioni programmatiche poste negli incipit e negli explicit) si segnala, negli ultimi anni, un maggiore ottimismo rispetto alle posizioni di Jæger, Nuyens, o anche di Balme. Senza voler discutere decine di autori e contributi, che spesso prendono posizione su questo o quel dettaglio dell'indice del corpus solo nella misura in cui la questione è rilevante per la loro esegesi delle dottrine dello Stagirita, si può osservare, come tendenza generale, una certa fiducia a 'prendere per buono' l'ordinamento del corpus a noi tramandato. Un buon esempio è il recente volume di Carlo $\mathrm{Natali}^{40}$, il quale - proprio perché informato da finalità quasi divulgative - non entra nelle minuzie dell'argomentazione e ci offre un 'precipitato' di quel che oggi quasi tutti assumono nel momento in cui si apprestano a interpretare il corpus psicobiologico: l'ordine di lettura suggerito dalla rete dei richiami incrociati non rispecchierebbe l'ordine cronologico della stesura, ma comunque sarebbe stato escogitato dall'autore, a scopo didattico ${ }^{41}$.

${ }^{39}$ M. Furth, Aristotle's Biological Universe: an Overview, in Philosophical Issues in Aristotle's Biology, a cura di A. Gotthelf, J.G. Lennox, Cambridge-New York, Cambridge University Press, 1987, pp. 21-52.

${ }^{40}$ C. Natali, Aristotele, Roma, Carocci, 2014. La biologia è trattata sotto la rubrica generale de Lo studio del mondo fisico (pp. 85-188), e in particolare alle pp. 139-166 (La fisica della natura vivente).

${ }^{41}$ Cfr. Natali, Aristotele, cit., p. 141. Di tanto in tanto, però, anche Natali avanza qualche dubbio circa il fatto che la ripulitura dell'opera sia stata perfettamente compiuta, come quando osserva che il $G A$ fa talora sorgere il sospetto di una doppia redazione (cfr. p. 159). 
E il perno fondamentale di tale ordinamento è la distinzione tra esposizione dei 'fatti' che troviamo nella Historia animalium ed esposizione delle cause, che troviamo nel Departibus e nel De generatione. Quanto Aristotele scrive in $H A$ I 6, 491a7-14, sembra volerci suggerire questo orientamento:

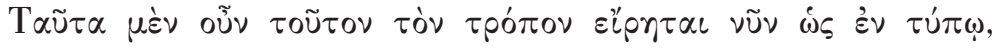

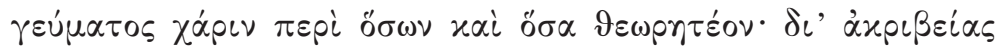

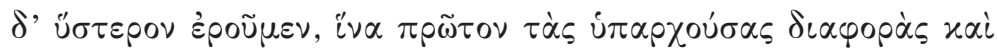

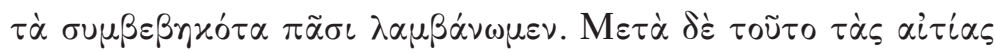

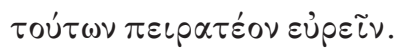

Queste cose [sc. l'introduzione generale alla Historia fornita nei capp. precedenti] sono state ora dette in questo modo come in abbozzo, per dare un assaggio circa le varie cose che bisogna indagare: in seguito ne parleremo con precisione, affinché anzitutto afferriamo le differenze esistenti [tra i vari animali] e gli accidenti di tutti [gli animali]. Dopo di ciò, bisognerà tentare di trovare le cause di queste cose. (Historia animalium, I 6, 491a714, trad. mia).

Dunque, stando al proposito qui esplicitato, la Historia esporrebbe i fatti, il "che", mentre $P A$ e $G A$ (e $I A$ ) esporrebbero le cause dei fatti, il "perché"‘2.

Come però è stato osservato da numerosi interpreti, $i$ "fatti" esposti in $H A$ non vanno concepiti come un mero centone di osservazioni 'pure': oltre al fatto che le osservazioni sono sempre (in Aristotele come in ogni altro scienziato) guidate da un'intenzione teorica, vi è da notare, nel caso particolare di $H A$, che tale intenzione teorica è esplicita; per giunta, le osservazioni vi sono catalogate in base a quattro grandi rubriche, stabilite secondo un criterio sulla cui scelta Aristotele si interroga consapevolmente:

${ }^{42}$ Cfr. Natali, Aristotele, cit., p. 149 per il rapporto tra $P A$ e $H A$, e pp. 160 e 162 per quello tra $H A$ e $I A$; quanto a $G A$, si tratterebbe del naturale completamento di $P A$ (cfr. p. 142 , dove si cita $G A 715$ a1-18, e p. 154), e di conseguenza starebbe, con $H A$, in un rapporto analogo a quello in cui si trova $P A$ (lo studio delle cause, 782a22, cfr. p. 159). 
dopo aver discusso, appunto, tale questione (HA I 1-6), da $H A$ I 7 fino a tutto l'intero libro IV si estende la descrizione ragionata delle differenze tra le parti organiche ( $\mu$ óp८ $\alpha$ ), nei libri V-VIII sono descritte le differenze tra le "operazioni vitali" ( $\pi \rho \alpha \dot{\xi} \xi \iota \iota, \beta \grave{\iota} \circ \iota)$, mentre il libro IX discute le "abitudini" $\left(\eta^{\prime} \vartheta \eta\right)$ degli animali ${ }^{43}$.

Vediamo adesso quali sono le ricadute di queste considerazioni sullo status di quel (particolarissimo) testo che è il corpus biologico.

\section{Che tipo di testo è il corpus aristotelico?}

Nell'iniziare a lavorare su questo problema, non avevo alcuna opinione consolidata circa tutte le questioni che finora abbiamo affrontato. Ma mi sono convinto via via che gli indizi relativi alle discrepanze di contenuto tra le diverse opere, che gli interpreti usano per valutare l'anteriorità o la posteriorità di un'opera rispetto a un'altra, sono spesso (anzi, direi quasi sempre) interpretabili sia nel modo voluto dall'interprete, sia in modo specularmente opposto. Un ottimo esempio sono i casi in cui la Historia presenta una forma 'compatta', o addirittura dà solo un accenno, di descrizioni che nel Departibus si trovano in forma estesa:

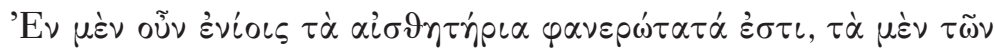

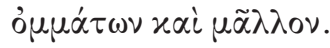

In alcuni [animali] gli organi di senso sono evidentissimi, e soprattutto quelli degli occhi. (Historia animalium IV, 8, 533a19-20, trad. mia).

\footnotetext{
${ }^{43}$ Questa la ricostruzione dell'indice della Historia animalium fornita da G.E.R. Lloyd, Aspects of the Relationship between Aristotle's Psychology and his Zoology, in Essays on Aristotle's De Anima, a cura di M.C. Nussbaum, A.O. Rorty, Oxford, Clarendon Press, 1992, pp. 147167 (cfr. in particolare p. 155); ristampato con qualche adattamento come The Relationship of psychology to zoology, in G.E.R. Lloyd Aristotelian Explorations, Cambridge-New York, Cambridge University Press, 1996, pp. 38-66.
} 
E qui finisce quel che Aristotele, nella Historia, ha da dire sugli occhi. La corrispondente trattazione, nel De partibus, comprende l'intero capitolo II 13, per un totale di una colonna e mezza circa dell'edizione Bekker ${ }^{44}$.

Cos'è avvenuto, qui? che Aristotele ha espanso, in $P A$, materiali osservativi precedentemente stoccati in forma più schematica in $H A$ (ipotesi standard, rappresentata p.es. da Natali)? oppure che $H A$ riassume le informazioni proprio perché $P A$ le aveva già fornite in maniera più dettagliata (ipotesi Balme)?

La questione è chiaramente indecidibile.

L'idea che $H A$ esponga il 'che', mentre $P A, I A$ e $G A$ ricerchino il 'perché', è evidentemente da prendere con la massima serietà, visto che è quella ufficiale di Aristotele; ma, come già detto qui e come più volte osservano Vegetti e Lanza, la Historia non può certo considerarsi come una mera collezione di osservazioni, e non è certo priva di indagini eziologiche: quindi anche qui c’è qualcosa di non chiaro.

Presenterò adesso qualche mia osservazione, sulla base dell'idea di Furth, già menzionata, che i libri centrali della Metafisica siano una discussione sul che cos'è di un organismo, e dell'osservazione di Balme, secondo cui il De generatione animalium prende partito a favore dell'esistenza delle forme sub-specifiche, idea che né in De partibus animalium né in Metaph. VIIIX è ancora articolata (nel migliore dei casi, è presentata come un qualcosa di problematico). Si assuma che $G A$ sia l'ultima (per i motivi detti) tra le opere biologiche; si può poi ipotizzare (come è ampiamente accettato dagli studiosi) che Metaph. VII-IX e $P A$ appartengano ad un unico periodo, anteriore a quello di $G A$. In questo periodo Aristotele non ha ancora accettato come pacifica l'esistenza di forme a livello sub-specifico: questo periodo però non può essere (come vorrebbe Balme) quello tardo-accademico, perché $P A$ presuppone un corpus di conoscenze che non è (a differenza di ciò che Balme

\footnotetext{
${ }^{44}$ In compenso, subito dopo il brevissimo accenno alla vista, la Historia si addentra, senza in alcun modo avvertire il lettore che verrà introdotta una digressione, in una minuta discussione sulle esperienze dei pescatori che dimostrano che i pesci hanno il senso dell'olfatto. Sono giunture come queste che conferiscono alla Historia il suo tipico aspetto da work-in-progress.
} 
pensa) quello di una normale persona colta, ma che richiede (almeno una parte sostanziale del)le osservazioni raccolte in $H A$.

Si dovrà dunque tornare al vecchio schema di successione $H A-P A-G A$, con $H A$ a funger da punto di riferimento databile grazie al suo ancoramento al periodo dei viaggi?

Non necessariamente, se diamo il debito peso ai fatti evidenziati da Balme: che solo una parte delle osservazioni contenute in $H A$ sono sfruttate in $P A$, e che proprio alcune delle osservazioni contenute in $H A$ potrebbero aver contribuito a portare dal paradigma di $P A$ a quello di $G A$ e alla scoperta delle varietà sub-specifiche.

In altri termini, io difendo (con la communis opinio) l'idea che la Historia animalium sia la grande raccolta di materiali dalla quale Aristotele ha tratto la maggior parte dei dati contenuti nelle altre opere, ma con i seguenti caveat: Aristotele (a differenza di quanto pensano Vegetti e Lanza) non ha 'degradato' $H A$ dal livello di trattato a quello di raccolta di materiali quando la sua ontologia è cambiata, ma ha da subito concepito $H A$ come tronco principale di cui tutti gli altri trattati biologici sono i rami; e (soprattutto) non ha mai smesso di arricchire $H A$, la cui stesura si deve dunque concepire come una concrezione progressiva fermatasi solo con la morte dell'autore.

Non difendo, quindi, l'idea che $H A$ fosse una raccolta di materiali strettamente ancillare alla stesura dei due trattati teorici (e di quelli soli): basti pensare a tutti gli spunti sull'etologia degli animali, che non trovano sviluppo nei trattati teorici ${ }^{45}$; ma non difendo nemmeno l'idea che $H A$ (come vogliono Vegetti e Lanza) rappresenti uno stadio precoce della scienza aristotelica, anteriore e addirittura alternativo a quello di $P A$ e $G A$. Il testo della Historia animalium corrisponde, a mio avviso, al risultato

\footnotetext{
${ }^{45}$ Che Aristotele avesse in mente un'opera di etologia, analoga a ciò che $P A$ è per l'anatomia e $G A$ per l'embriologia? È lecito supporre che il De motu animalium fosse il I libro di quest'opera, che ne esponeva l'architettura eziologica, più o meno come fa $P A$ I per l'insieme del Departibus?
} 
dell'ultimo stadio di riordinamento degli appunti personali di Aristotele biologo. Stadio necessariamente provvisorio, come provvisorio è ogni workin-progress, e dunque approssimativo: così si spiegherebbero le incongruenze di contenuto, le descrizioni e le 'schede' a volte incompiute, le intromissioni di determinate osservazioni in contesti inappropriati se valutati dal punto di vista sistematico; così si potrebbero (forse) spiegare persino i contenuti dei due ultimi libri, i cui tratti non ortodossi sono stati notati da più studiosi: potrebbe trattarsi di raccolte di informazioni che Aristotele non avrebbe avuto il tempo di vagliare ed emendare.

Che poi questa raccolta di materiali possa anche essere stata ampliata da successori di Aristotele, o che Aristotele stesso possa aver delegato la raccolta dei materiali a vari collaboratori, che cioè $H A$ possa avvicinarsi allo statuto di opera ad autorialità collettiva (e che ciò possa valere in particolare per i due ultimi libri), è un fatto aggiuntivo, che può integrarsi con l'ipotesi che ora ho delineato.

\section{Rapporto trail De anima e il corpus biologico}

Potremmo dire, in estrema sintesi, che nelle opere biologiche Aristotele tratta della natura animale da due punti di vista, il punto di vista delle parti e quello delle funzioni; i due punti di vista sono quasi sempre integrati tra loro; e ciò, in conformità con l'idea fondamentale dell'anatomia funzionale tipica dello Stagirita, in base alla quale la parte è "in vista" della funzione che svolge ed è da essa definita ${ }^{46}$ :

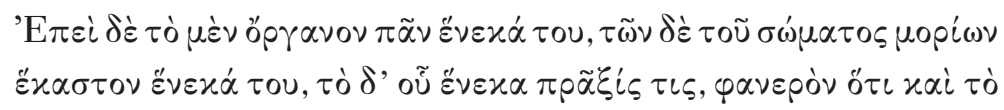

\footnotetext{
${ }^{46} \mathrm{Il}$ principio è enunciato chiaramente in de An. II 1, 412a27-b1. Una nota conseguenza di questo postulato (noto come "principio di ominimia") viene esplicitata in Mete. IV 12, 390a10-20: ciò che non è più in grado di svolgere la propria funzione perde la propria essenza; per l'applicazione di questo principio alle parti organiche, cfr. in particolare le rr. 11-12: l'occhio è davvero tale solo se capace di servire alla funzione visiva.
} 


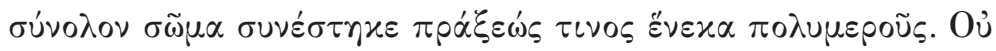

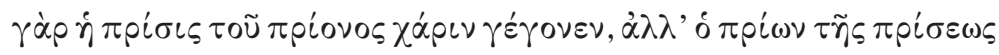

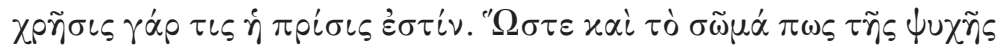

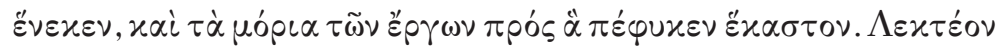

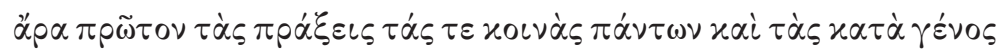

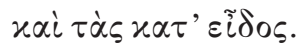

E poiché ogni strumento è in vista di qualcosa, e poiché ciascuna delle parti del corpo è in vista di qualcosa, e ciò in vista di cui [è] è una qualche azione, è chiaro che anche il corpo intero è costituito di molte parti in vista di qualcosa. Non è infatti l'atto di segare a essersi generato in vista della sega, ma la sega in vista dell'atto di segare. Sicché anche il corpo in qualche modo è in vista dell'anima, e le parti [del corpo] sono in vista delle operazioni per le quali ciascuna è naturalmente costituita. Bisogna dunque parlare anzitutto delle azioni: quelle comuni a tutti i viventi, quelle che si estendono su interi generi, e quelle proprie delle specie. (Departibus animalium, I 5, 645b14-22, trad. mia).

Il fatto che la parte sia in vista della funzione comporta che la funzione sia logicamente anteriore alla parte. Da questo punto di vista, risulta ovvia l'anteriorità logica della trattazione sulla funzione del vivere considerata da un punto di vista generale (il De anima, che tratta delle funzioni comuni a tutti i viventi, e poi i Parva naturalia, che trattano anche di funzioni come la respirazione, che si estendono su singoli macrogeneri) rispetto alle specifiche funzioni fisiologiche, nonché alle strutture corporee in cui la vita si realizza; tanto più che Aristotele specifica che tra la funzione generale di vivere e il corpo intero vige lo stesso rapporto che vige tra le singole funzioni e le singole parti del corpo. Sembra dunque che la consecuzione De anima-Parva naturalia - scritti biologici rispetti e rispecchi i seguenti principii: (a) la definizione generale dell'anima deve precedere quelle delle sue singole parti, in quanto queste specificano la prima (sia l'una sia le altre sono incluse nel De anima); (b) la definizione delle parti dell'anima deve essere seguita da una 
trattazione più articolata delle loro funzioni specifiche (Parva naturalia); (c) una volta giunti a un certo livello di dettaglio, è inevitabile trattare insieme delle parti e delle funzioni (corpus biologico $)^{47}$.

\section{Conclusioni}

È ora il momento di tirare delle conclusioni.

Se dobbiamo prestar fede ai racconti di Strabone e Porfirio, non solo non sarebbe stato Aristotele a conferire al corpus (nè al corpus nel suo insieme nè a quella particolare frazione di esso che è il corpus biologico) l'ordinamento a noi tramandato, ma questo avrebbe subito vicissitudini materiali di ogni tipo, prima che i singoli trattati assumessero la facies che ci è familiare. In tal caso, a mio avviso, non esisterebbe un criterio per ricostruirne la facies originaria. Per quanto riguarda i rinvii incrociati che spesso troviamo tra le diverse opere, quasi mai essi sono così integrati nella sintassi del testo da permettere di escludere che si tratti di inserzioni posteriori. Occorrerebbe un esame dettagliato di ciascun singolo rinvio, e valutare, caso per caso, se il rinvio è effettivamente funzionale all'argomentazione che lì viene svolta, o se invece si tratta di un'inserzione che può essere espunta senza pregiudizio per la trattazione; onde selezionare solo quei rinvii che in tal modo avremmo scoperto essere stati indubitabilmente operati da Aristotele; e quindi, sulla base dei rinvii in tal modo selezionati, costruire la mappa globale dei

${ }_{77}$ Trovo dunque convincente la prospettiva di S. Menn, Aristotle's Definition of Soul and the Programme of the 'De Anima', in «Oxford Studies in Ancient Philosophy», XXIII (2002), pp. 83-139: la definizione generale dell'anima data in De anima II 1 sarebbe a suo avviso «a criterion for judging his own [sc. Aristotle's] account of what the soul is» (p. 103); i libri II e III fornirebbero appunto questo «account». Menn prosegue (p. 105) sottolineando che la principale conseguenza di definire l'anima come «atto primo» è quella di sottolineare la sua natura di disposizione a un'attività; la specificazione di quali siano le attività per le quali l'anima è disposizione occuperebbe (nell'ordinamento proposto da Menn) l'intero blocco De anima-De partibus animalium, visto che questo trattato si occupa degli organi di cui si serve l'anima per svolgere le sue funzioni (cfr. p. 107). Non trovo però che il ragionamento debba limitarsi al De partibus, visto che può applicarsi altrettanto bene ai Parva naturalia, al De animalium incessu e al De generatione animalium. 
riferimenti intertestuali, per poi capirne la logica complessiva. Precisamente a questa tipologia, per noi più pertinente, appartiene, mi sembra, la 'dichiarazione programmatica' in $H A$ I 6, 491a7-14 sopra citata ${ }^{48}$, in cui ci viene detto che la Historia animalium deve precedere $P A$ e $G A$.

D'altro canto, vi è da tener conto di un secondo fatto, che limita l'importanza di queste vicissitudini ai fini del lavoro esegetico.

Sappiamo che le opere che stiamo considerando erano 'esoteriche': non destinate alla pubblicazione. Esse sono dunque rimaste presso l'autore fino alla morte di questi; è quindi più che plausibile ipotizzare (come è del resto ormai pressoché communis opinio degli studiosi di Aristotele) che siano state soggette a una revisione continua, nel corso della quale ciascuna opera ha influito sull'elaborazione di ogni altra. Ora, se è vero che di una nozione come quella di “opera” (scientifica, filosofica, letteraria, eccetera) si può misurare la validità solo in quanto tale nozione ci aiuta nella comprensione delle singole opere che ci proponiamo di studiare, è forse lecito chiedersi se per caso, per il corpus di Aristotele, non avremmo bisogno di un concetto di 'opera' diverso da quello utilizzato, p.es., per Platone. Ciò che va esclusa è l'idea di una trattazione continua da leggersi dall'inizio alla fine, dall'incipit delle Categorie all'explicit della Poetica. Alla luce di ciò che abbiamo detto, va anche esclusa l'idea di una serie di trattazioni ciascuna in sé conclusa, ma indipendenti l'una dall'altra.

Forse l'idea che meglio ci può aiutare, sia (di nuovo) per il Corpus Aristotelicum nel suo insieme sia specificamente per il corpus biologico, è quella di un macrotesto costituito come una rete, in cui ciascun singolo passo (ciascun singolo nodo della rete) richiede di essere confrontato con tutti gli altri. In questa grande 'rete', sarà non di meno possibile individuare dei continua dotati di una propria relativa autonomia. In genere queste trattazioni coincidono non con quelle che ci sono state tramandate come singole opere, bensì con singoli libri o con singoli gruppi di libri: p.es. De

\footnotetext{
${ }^{48}$ Cfr. supra, p.
} 
anima II-III. In certi casi, viceversa, coincidono con insiemi di opere: è il caso del blocco costituito dal De anima e da alcuni trattatelli compresi nei Parva naturalia che implementano trattazioni del De anima (penso p.es. al De sensu, al De somno et vigilia e al De insomniis, che affrontano in maniera più estesa e compiuta tematiche che il trattato principale aveva lasciato in sospeso, come il problema della sensazione comune e quello della natura dell'illusione sensoria e dell'errore percettivo). In altri casi ancora, Aristotele sembra aver voluto concentrare, in un unico breve trattato, una visione sintetica di questioni che nel resto del corpus biologico erano state trattate in più punti distinti: esempio tipico, il De motu animalium, che affronta al contempo problemi psicologici già trattati in De anima III 9-11 (la natura della orexis) e problemi di anatomia funzionale prossimi a quelli del $D e$ partibus (quali siano i requisiti anatomici minimi di un sistema semovente). In ogni caso, viste le vicissitudini del corpus, la decisione su quale sia il continuum in questione non può mai esser data per scontata, come se fosse data a monte del lavoro di interpretazione, ma è sempre parte integrante del lavoro dell'interprete.

Se si adotta questa impostazione, il danno costituito dalla peculiare trasmissione del corpus (vere o false che siano le testimonianze a noi tramandate) viene, almeno in parte, ridimensionato: quel che conta, è la presenza e la riconoscibilità di tali continua, non l'ordine in cui ci sono stati tramandati. 